\title{
A COLLISIONLESS SCENARIO FOR URANUS TILTING
}

\author{
GWenä̈L Boué, AND JACQUes LASKAR \\ Astronomie et Systèmes Dynamiques, IMCCE-CNRS UMR8028, Observatoire de Paris, UPMC, 77 Av. \\ Denfert-Rochereau, 75014 Paris, France \\ boue@imcce.fr
}

October 25, 2018

\begin{abstract}
The origin of the high inclination of Uranus' spin-axis (Uranus' obliquity) is one of the great unanswered questions about the Solar system. Giant planets are believed to form with nearly zero obliquity, and it has been shown that the present behaviour of Uranus' spin is essentially stable. Several attempts were made in order to solve this problem. Here we report numerical simulations showing that Uranus' axis can be tilted during the planetary migration, without the need of a giant impact, provided that the planet had an additional satellite and a temporary large inclination. This might have happened during the giant planet instability phase described in the Nice model. In our scenario, the satellite is ejected after the tilt by a close encounter at the end of the migration. This model can both explain Uranus' large obliquity and bring new constraints on the planet orbital evolution.
\end{abstract}

Subject headings: celestial mechanics — planets and satellites: general — solar system: general

\section{Introduction}

Today, Uranus' obliquity $\left(97^{\circ}\right)$ is essentially stable. This s'due to the regularity of its orbital motion and to the slow Pprecession motion of Uranus' axis compared to the secular frequencies of the Solar system (Laskar \& Robutel 1993). It is, sometimes believed that a great collision with an Earthsized protoplanet could be the reason of Uranus large obliquity. But this straightforward scenario hardly explains the presence of regular satellites orbiting Uranus in its equatorial plane (Korycansky et al. 1990). However, the presence of satellites around a planet can increase its precession rate of by a large amount depending on their mass and their orbital parameters (Tremaine 1991; Goldreich 1966; Ward 1975; Boué \& Laskar 2006). For example, with a satellite of mass $m=0.01 M_{U}$, where $M_{U}$ is the mass of Uranus, the - increase can reach a factor 1000 (Fig. 1). The maximal effect 7 is obtained for a satellite located at about 50 Uranian radii, which is actually in the region where a satellite has been predicted by some formation models (Mosqueira \& Estrada 2003a b; Estrada \& Mosqueira 2006). For comparison, the most distant regular satellite of Uranus is Oberon, its mass is $3.45 \times 10^{-5} M_{U}$, and its distance from Uranus' barycenter is 23 Uranian radii (Laskar \& Jacobson 1987). The interactions between spin-axes and secular motions of the planets are also strenghtened when orbital inclinations are high. Such conditions could be met during the planetary migration. Indeed, in the Nice scenario (Tsiganis et al. 2005), Jupiter and/or Saturn should have undergone close encounters with the ice giants to reach their present eccentricities (Morbidelli et al. 2009). These close encounters can raise the inclinations. Moreover, the additional satellite can be ejected during one of these encounters. We therefore propose that Uranus had an additional satellite and that its spin-axis was tilted during the planetary migration.

\section{Numerical experiment}

The construction of such a scenario for Uranus tilting can be described in four steps.

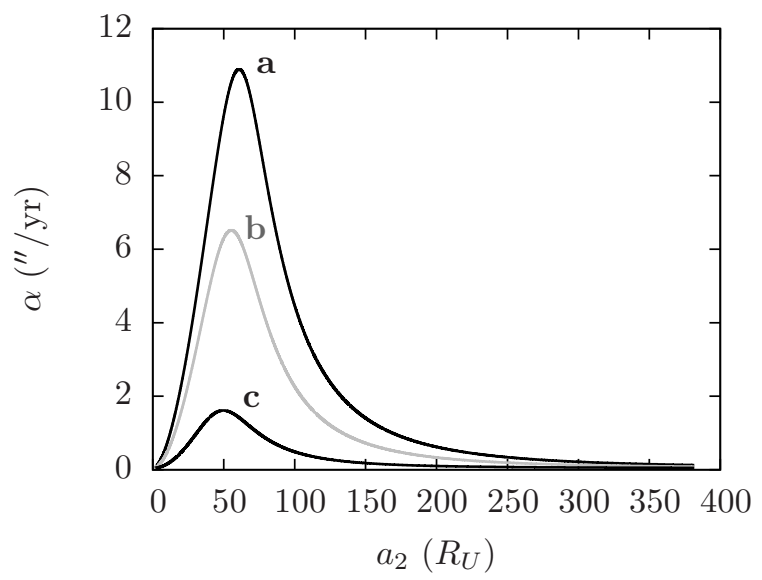

Fig. 1.- Uranus precession frequency in presence of a heavy satellite. Uranus effective precession constant as a function of the distance of an additional satellite of mass $m=0.01 M_{U}(\mathbf{a}), m=0.005 M_{U}(\mathbf{b})$, and $m=0.001 M_{U}$ (c), where $M_{U}$ is the mass of Uranus (Boué \& Laskar 2006). For this calculation, the semi-major axis and the eccentricity of Uranus are set to the current values, and the satellite is assumed to have a circular orbit. All inclinations as well as the obliquity are set to 0 .

First, we simulated the Nice model (Tsiganis et al. 2005). We integrated 10000 migrations of the giant planets over 10 


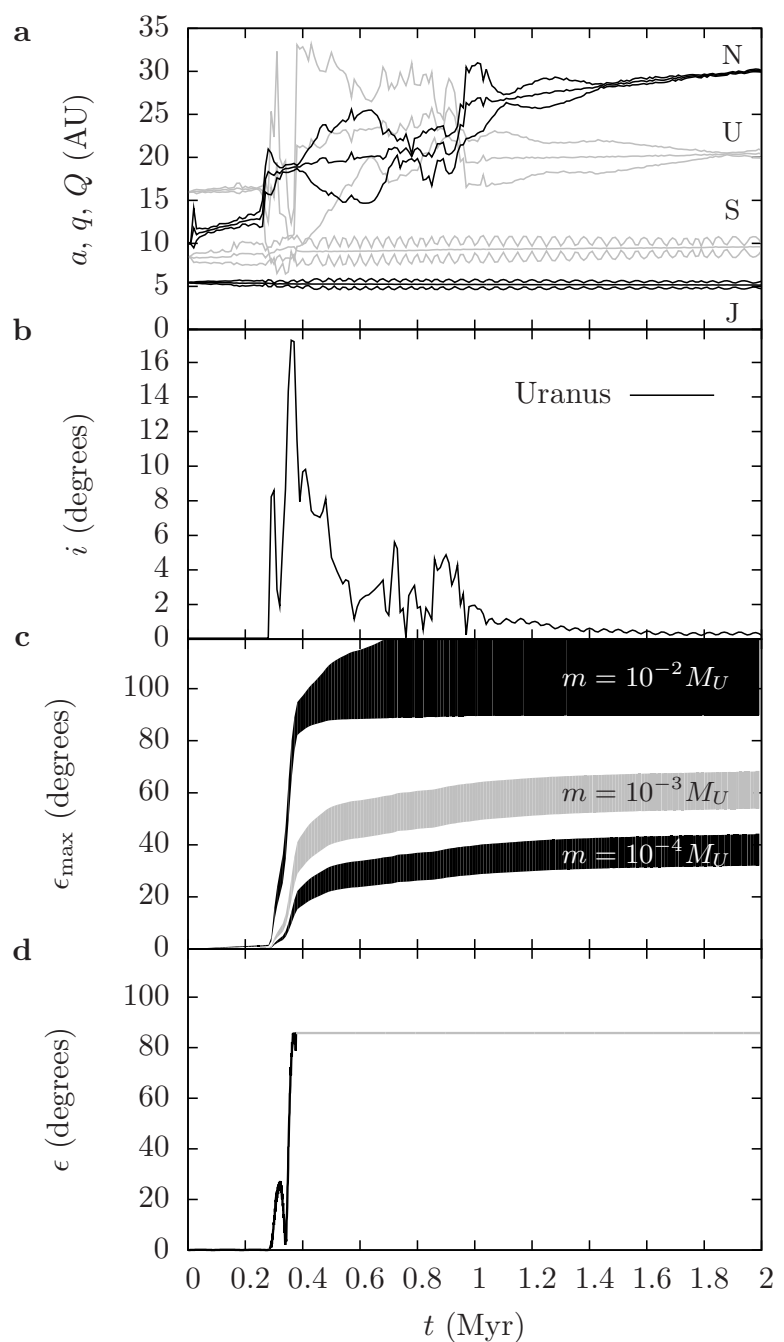

Fig. 2.- Comparison between orbital evolution and obliquity increase. a,b, Example of orbital evolution of the giant planets during the planetary migration over $2 \mathrm{Myr}$. (a) semi-major axis, minimum, and maximum heliocentric distances. (b) Uranus inclination. c, Maximal tilt starting from zero obliquity for any orbital evolution with the same semi-major axis, eccentricity and inclination as Uranus in subfigures $(\mathbf{a}, \mathbf{b})$. In this calculation (see Fig. [5), the effect of an additional satellite at 50 Uranian radii is implicitely taken into account in the precession constant. We considered three different masses for the satellite : $10^{-4} M_{U}, 10^{-3} M_{U}$, and $0.01 M_{U}$, where $M_{U}$ is the mass of Uranus. For each mass, the satellite eccentricity is set to 0 (lower boundary) and 0.5 (upper boundary). d, Results of a numerical integration with a satellite of mass $m=0.01 M_{U}$ (black curve). The satellite is ejected by a close encounter with Saturn at $t=0.38 \mathrm{Myr}$. Once the satellite is ejected, the obliquity remains constant (gray curve). In these plots, the obliquity is measured relative to the fixed plane orthogonal to the final total orbital angular momentum.
Myr. For these simulations,

$$
\begin{aligned}
\dot{a} & =\frac{\delta a}{\tau} \exp (-t / \tau) \\
d e / d t & =-e /\left(2 \tau_{e}\right) \\
d i / d t & =-i /\left(2 \tau_{i}\right)
\end{aligned}
$$

with $\tau=2 \mathrm{Myr}$, and $\tau_{e}=\tau_{i}=\tau / 10$ as in (Lee et al. 2007). $a$, $e$ and $i$ are respectively the semi-major axis, the eccentricity and the inclination of the giant planets; $t$ is the time and $\delta a$ is the difference between the initial semi-major axis of a planet and its current one. The initial semi-major axis of Jupiter was set to $5.45 \mathrm{AU}$. The initial semi-major axis of the other planets were obtained randomly with a uniform distribution. The initial semi-major axis of Saturn was varied in the range 8.38-8.48 AU. The initial order of the ice giants was modified compare to the current Solar system : the initial semi-major axis of Neptune was varied in the range 9.9-12 AU and the initial semi-major axis of Uranus was varied in the range 13.417.1 AU. The terrestrial planets are not taken into account in this study.

Then, out of the 5142 simulations that survived without ejection or planet collision, we selected those where the planet final order is the same as in the Solar system. We obtained 1995 different integrations. As the tilt requires high inclination, we kept only the simulations where Uranus' inclination increases beyond a given threshold. We set this threshold to $17^{\circ}$ which limits the number of simulations to 31 . Among these simulations, we rejected those where the closest encounter between Uranus and any other planet is closer than 50 Uranian radii. With our criterion, we finally selected 17 simulations. One of them is displayed in figure 2 $2 \mathrm{a}, 2 \mathrm{~b}$. We call it the reference simulation.

In a third step, we computed the maximal effect of an additional satellite on Uranus' obliquity in any orbital evolution with the same semi-major axis, eccentricity and inclination as in the reference simulation, regardless the conjugated angles. For that, we used the expression of the effective precession constant as a function of the satellite orbital parameters (Boué \& Laskar 2006). Then, we computed the maximal tilt given Uranus orbital evolution (Boué et al. 2009). In the calculations, the satellite is at 50 Uranian radii in both circular and eccentric orbits. Figure 2 r shows the maximal obliquity that has been reached in these simulations. The evolution shows clearly that the tilt can only occur when the inclination is high. In the present case, a satellite with $m=0.01 M_{U}$ is still necessary for the obliquity to reach $97^{\circ}$.

Finally, we integrated the evolution of Uranus' spin-axis and the additional satellite in the 17 selected simulations. Calculations of the evolution of Uranus' spin-axis take into account the gravitational torques exerted by the Sun, by the additional satellite and by all the other giant planets. For each of the 17 planet migrations, we performed 100 integrations varying the initial semi-major axis of the satellite by a small amount (15 meters). The final obliquity distribution is given in figure 3. In 644 cases, the obliquity does not exceed $10^{\circ}$ because the satellite is ejected at the first encounter before the increase of the inclinations. But, if the satellite survive the first encounter, as in $62 \%$ of the cases, then the obliquity can reach large values. Among the integrations in which the satellite is ejected before the end of the migration, there is a final obliquity larger than $60^{\circ}$ in 220 cases and an obliquity larger than $90^{\circ}$ in 37 cases. 


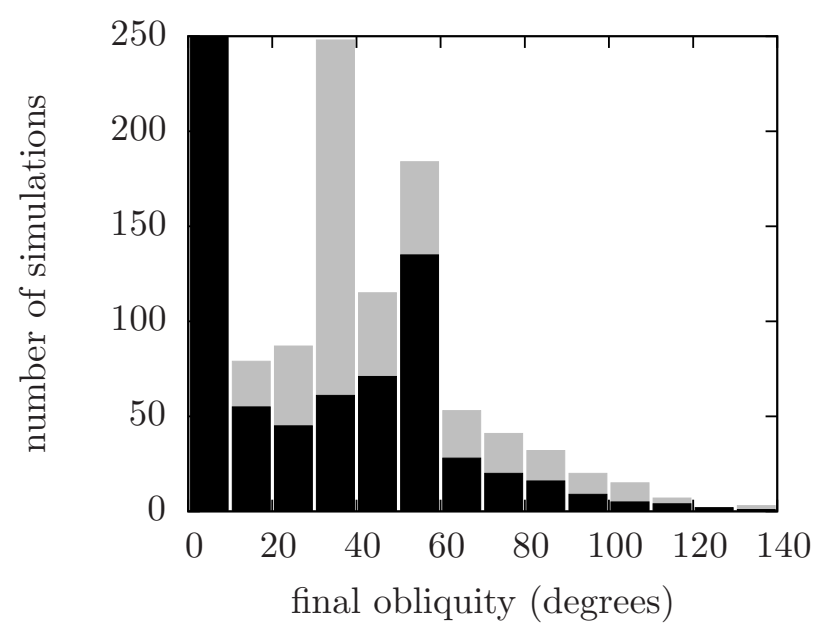

Fig. 3.- Distribution of Uranus final obliquity. This is the result of 1700 integrations of Uranus spin-axis with an additional satellite : 100 per each of the 17 selected migrations. In black, the cases where the satellite is ejected; in gray, the cases where the satellite still orbits Uranus after 2 Myr (the end of the integrations with a satellite). The first bin has been troncated for a better visualization, its value is 644 (black) +8 (gray). Among the simulations with ejection of the satellite, there is a final obliquity larger than $60^{\circ}$ (resp. $90^{\circ}$ ) in 220 cases (resp. 37 cases).

\section{Dynamics of the tilt}

Here we explain the evolution of Uranus' spin-axis during the tilt presented in the figure 2. The smooth evolution of the obliquity during the tilt (Fig. $4 \mathbf{b}$ ) suggests that it is due to a resonance. In the following, we show that the tilt actually occurs during a 1:1 spin-orbit resonance between the precession of Uranus' axis and the regression of the node of its orbit. The obliquity $\epsilon$ is measured relative to the invariant plane orthogonal to the total orbital angular momentum at the end of the simulation (10 Myr). Traditionally, it is defined relative to the orbital plane, but as the inclination rises to high values, it is preferable to use the invariant plane in order to avoid artificial evolution of the spin-axis. At the end of the reference simulation, Uranus' orbital inclination is very small $\left(0.0024^{\circ}\right)$ and the difference between the two definitions is sufficiently small to be neglected. Let $(\boldsymbol{i}, \boldsymbol{j}, \boldsymbol{k})$ be a base frame such that the $x-y$ plane coincides with this invariant plane. We note $\boldsymbol{w}$, Uranus' spin-axis, and $\boldsymbol{n}$, the normal of its orbit. The obliquity $\epsilon$ is thus defined by $\cos \epsilon=\boldsymbol{k} \cdot \boldsymbol{w}$. Let $\phi_{\alpha}$ and $\phi_{\nu}$ be the angles measured positively from the reference direction $\boldsymbol{i}$ to the projections of $\boldsymbol{w}$ and $\boldsymbol{n}$ into the $x-y$ plane, respectively. The evolution of $\psi=\phi_{\alpha}-\phi_{\nu}-\pi$ is not steady but describes plateaus during the tilt (Fig. $4 \mathbf{a}$, phases $I I$, and $I V+V)$. This confirms the $1: 1$ resonance between the precession of Uranus' axis and the regression of the node of its orbit.

In order to have a full understanding of the tilt, we now give the equations of motion that will allow us to describe the spatial evolution of the spin-axis displayed in the figure $4 \mathrm{~d}$.
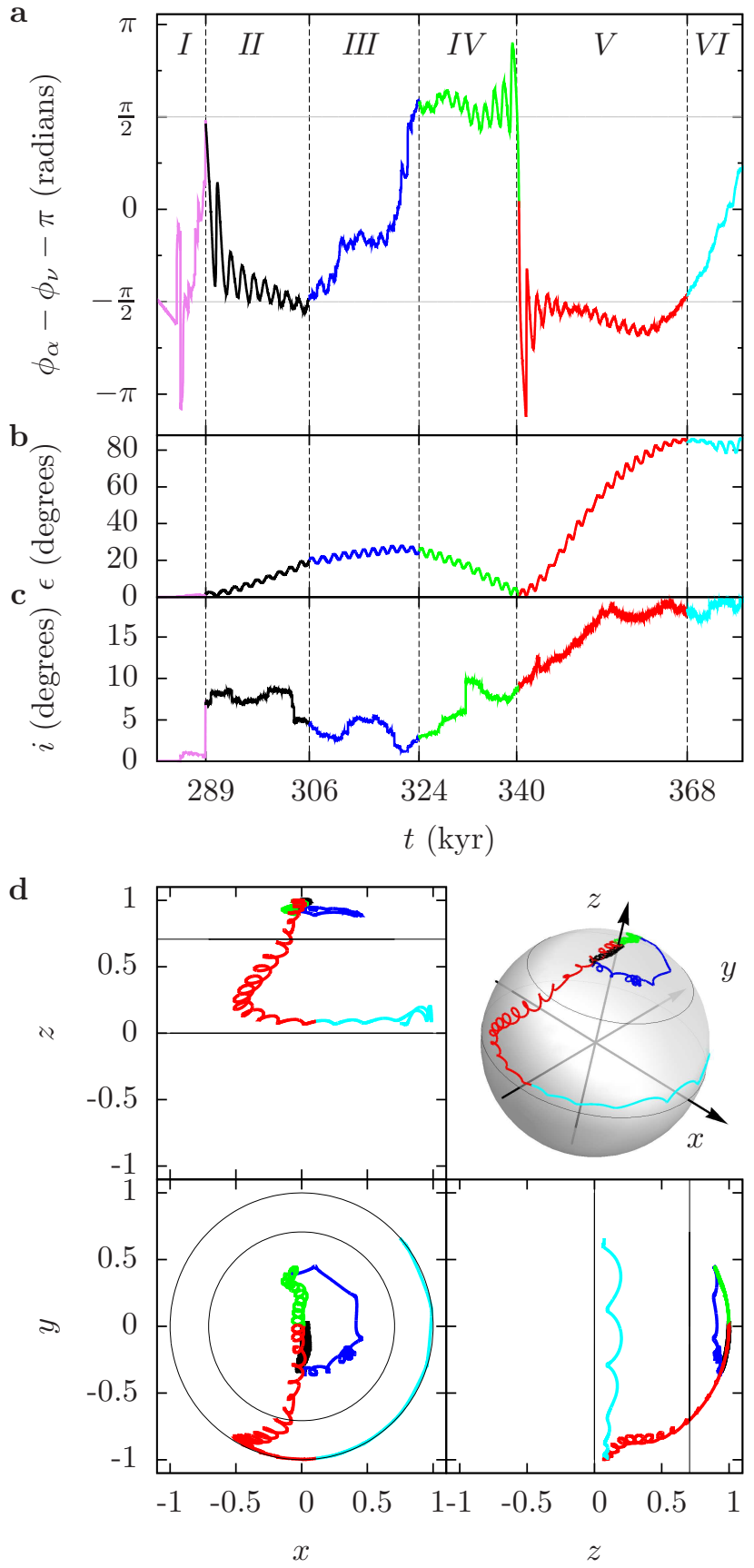

Fig. 4.- Details on Uranus tilting. a, Evolution of the resonant angle $\psi=\phi_{\alpha}-\phi_{\nu}-\pi$ where $\phi_{\alpha}$ and $\phi_{\nu}$ are angles measured positively from a reference direction to the projection of Uranus' spin-axis $\boldsymbol{w}$ and Uranus' orbit pole $\boldsymbol{n}$ into the $x-y$ plane, respectively. $\mathbf{b}$, Evolution of Uranus' obliquity relative to the invariant plane. c, Evolution of Uranus' orbital inclination. d, Evolution of Uranus' spin-axis. The coordinates are $x=\sin \epsilon \cos \psi, y=\sin \epsilon \sin \psi$, and $z=\cos \epsilon$. The thin black circles in the $x-y$ plane, that correspond to the thin black lines in the $x-z$ plane and in the $y-z$ plane, represent the locations where the obliquity is $45^{\circ}$ (inner circle), and $90^{\circ}$ (outer circle). Uranus tilting is characterized by three resonant phases labelled $I I$, and $I V+V$ separated by non-resonant evolutions labelled $I, I I I$, and $V I$. 
of the satellites, and $\nu$ the regression frequency of Uranus' orbital node. In the frame rotating around $\boldsymbol{k}$ with the regression frequency $\nu$, the Hamiltonian describing the spin-axis evolution reads

$$
H=-\frac{\alpha}{2}(\boldsymbol{n} \cdot \boldsymbol{w})^{2}-\nu(\boldsymbol{k} \cdot \boldsymbol{w}) .
$$

In this frame $\boldsymbol{k}$ is constant, whereas $\boldsymbol{n}$ varies due to the evolution of the inclination. We have

$$
\boldsymbol{k}=\left(\begin{array}{l}
0 \\
0 \\
1
\end{array}\right), \quad \boldsymbol{n}=\left(\begin{array}{c}
-\sin i \\
0 \\
\cos i
\end{array}\right), \quad \text { and } \quad \boldsymbol{w}=\left(\begin{array}{l}
x \\
y \\
z
\end{array}\right)
$$

with $x=\sin \epsilon \cos \psi, y=\sin \epsilon \sin \psi$, and $z=\cos \epsilon$. One can go from these variables to Ward \& Hamilton (2004) ones by a rotation of angle $i$ around the second axis. The equations of motion associated to the Hamiltonian (1) are given by

$$
\frac{d \boldsymbol{w}}{d t}=\nabla_{\boldsymbol{w}} H \times \boldsymbol{w}
$$

which gives

$$
\frac{d \boldsymbol{w}}{d t}=-\alpha(\boldsymbol{n} \cdot \boldsymbol{w}) \boldsymbol{n} \times \boldsymbol{w}-\nu \boldsymbol{k} \times \boldsymbol{w}
$$

or equivalently

$$
\left\{\begin{array}{l}
\dot{x}=\omega_{z} y \\
\dot{y}=-\omega_{z} x-\omega_{x} z \\
\dot{z}=\omega_{x} y
\end{array}\right.
$$

with

$$
\begin{aligned}
& \omega_{z}=\alpha \cos i(z \cos i-x \sin i)+\nu, \\
& \omega_{x}=\alpha \sin i(z \cos i-x \sin i) .
\end{aligned}
$$

These equations are a combination of two rotations. The first one is a rotation around the third axis with the angular velocity $\omega_{z}$. The second is a rotation around the first axis with the angular velocity $\omega_{x}$.

Now we describe the evolution of Uranus' spin-axis displayed in the figure 4 .

During phase $I$, from $t=0$ to $t=289 \mathrm{kyr}$, the inclination $i$ and the obliquity $\epsilon$ are small, the three axes $\boldsymbol{w}, \boldsymbol{n}$, and $\boldsymbol{k}$ are almost aligned, there is no evolution.

In phase $I I$, the variable $\psi$ remains close to $-\pi / 2$ (Fig. 4a), there is thus a 1:1 spin-orbit resonance. In that case, the angular velocity $\omega_{z}$ in (5, 6) is negligible and it remains only the rotation around the first axis

$$
\left\{\begin{array}{l}
\dot{x} \approx 0 \\
\dot{y} \approx-\omega_{x} z \\
\dot{z} \approx \omega_{x} y
\end{array}\right.
$$

As the axis $\boldsymbol{w}$ describes an arc of a circle in the $y$ - $z$ plane (Fig. 4 $\mathbf{d}$ ), the third component $z$ decreases, and thus the obliquity $\epsilon$ increases (Fig. 4b).

In phase III, the angle $\psi$ evolves (Fig. 4a), hence the resonance is broken and the angular velocity $\omega_{z}$ becomes important. On the other side, the inclination decreases (Fig. 4c). Thus, the angular velocity $\omega_{x} \propto \sin i$ decreases too. Hence, the rotation in the $x-y$ plane dominates (Fig. $4 \mathrm{~d}$ )

$$
\left\{\begin{array}{l}
\dot{x} \approx \omega_{z} y \\
\dot{y} \approx-\omega_{z} x \\
\dot{z} \approx 0
\end{array}\right.
$$
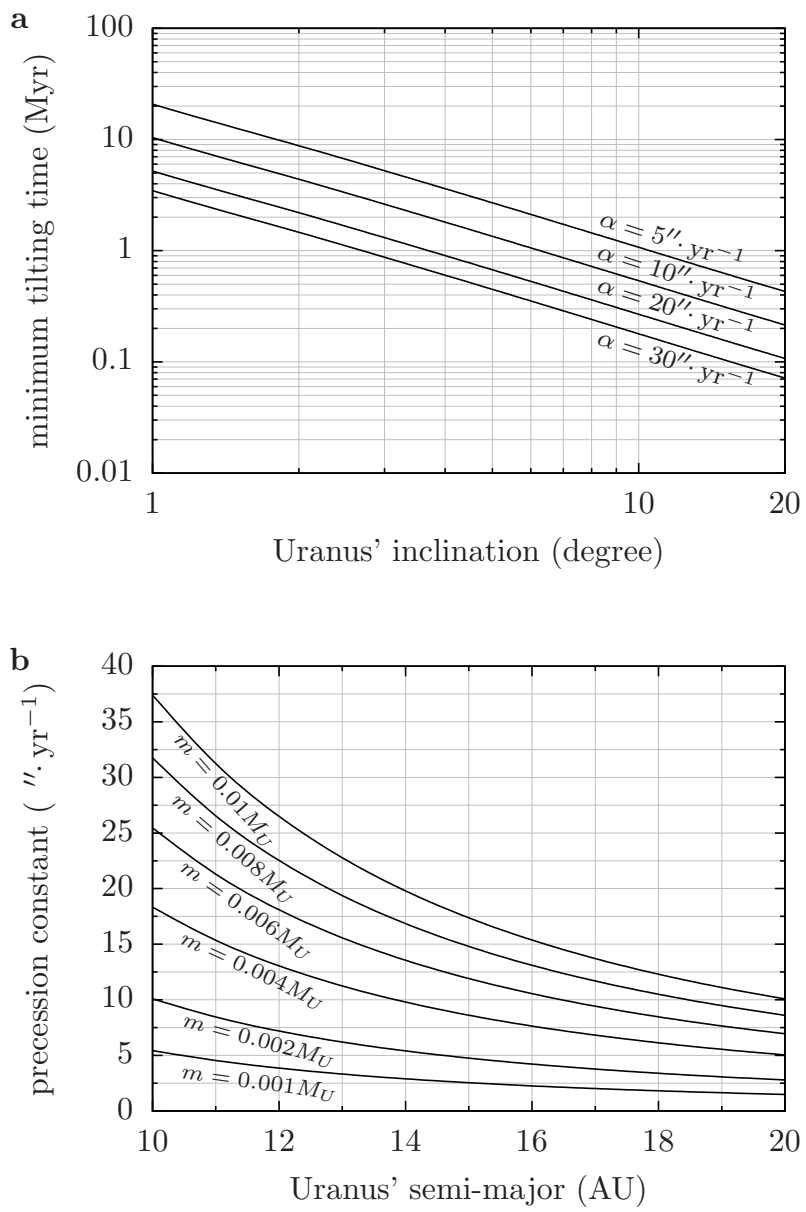

Fig. 5.- Constraints on the migration and on the mass of the satellite. a, Lower boundary on the time required to tilt Uranus by $97^{\circ}$ as a function of the inclination of its orbit for different precession constants (Boué et al. 2009). b, Uranus' precession constant as a function of its semi-major axis for different masses of the satellite in circular orbit (Boué \& Laskar 2006). In case of eccentric orbit of the planet, the semi-major axis $a_{U}$ should be replaced by $a_{U} \sqrt{1-e_{U}^{2}}$. For example, if during the planetary migration, Uranus' inclination remains above $10^{\circ}$ at least 1 Myr, then the precession constant should be larger or equal to $5^{\prime \prime} \cdot \mathrm{yr}^{-1}$ to tilt Uranus (a). Such a precession constant can be reached with a satellite of mass $m=0.001 M_{U}$ if $a_{U} \sqrt{1-e_{U}^{2}}$ is less than $10 \mathrm{AU}$ during the period of large inclination (b). 
and the obliquity $\epsilon$ is almost stationary (Fig. 4b).

During phase $I V$, the angle $\psi$ is stable for a second time. The spin-axis is thus once again captured in resonance and the equations of motion are the equations (7). The axis thus describes an arc of a circle in the $y$ - $z$ plane (Fig. $4 \mathrm{~d}$ ). This time, it starts with $y>0$ and $z<1(\psi \approx \pi / 2)$ and goes toward $y=0$ and $z=1$. As $z$ increases, the obliquity decreases (Fig. 4b). The orbital inclination $i$ is similar in phase $I I$, and $I V$ (Fig. 4le), so is the angular velocity $\omega_{x}$.

When the spin-axis crosses the $x$ - $z$ plane, the angle $\psi$ jumps from $\pi / 2$ to $-\pi / 2$ and the system enters in phase $V$ (Fig. 4a). The evolution is similar to the one of phase $I I$. The spin-axis describe an arc of a circle in the $y$ - $z$ plane on the $y<0$ side (Fig. 4 d). The obliquity increases (Fig. 4b). However, as the inclination is higher than in phase $I I$ $\left(18^{\circ}\right.$, see Fig. 4c) , the angular velocity $\omega_{x}$ is larger and the obliquity evolves faster (Fig. 4b).

In phase $V I$, the resonance is once again broken (Fig. 4a), the angular velocity $\omega_{x}$ becomes negligible with respect to $\omega_{z}$. The spin-axis describes an arc of a circle in the $x-y$ plane (Fig. 4 d), and the obliquity remains constant (Fig. 4b).

After the tilt, the satellite is ejected. The precession constant $\alpha$ decreases by a factor close to 1000 , which gives $\omega_{z} \approx \nu$ and $\omega_{x} \approx 0$. The equations of motion (5) thus show that the spin-axis precesses around the third axis at the angular velocity $\nu$. The obliquity remains constant as one can see in the figure 2 .

\section{Conclusion}

We have shown that the current obliquity of Uranus is compatible with planetary formation scenarios predicting small initial obliquities without the need of a large collision. Moreover, we confirm the necessity of the close encounters used in the Nice model to recover the present eccentricity of Jupiter and Saturn. Additionally, we solve the problem of the missing satellite around Uranus (Mosqueira \& Estrada $2003 \mathrm{a}, \mathrm{b}$ ). Although stellite formation theories are not at the stage that they can constrain the final mass of the satellites, we acknoledge that the satellite we have introduced may be too massive. Nevertheless, a less massive satellite of only $0.001 M_{U}$ can still be sufficient to tilt Uranus if the planetary migration timescale is larger than the one used here (see figure 5). Several recent studies actually suggest such a longer migration timescale (Murray-Clay \& Chiang 2005; Boué et al. 2009; Lykawka et al. 2009). The parameters involved in the formation of the Solar system are still too numerous to be able to derive precise estimates of the probability of the present scenario to occur, but with this additional satellite, we are able to propose a scenario that fits with the present late migration scenario as given by the Nice model. Depending on the progress made in satellite formation theories and possible future variations in the migration scenarios, the elements given here could provide strong additional constraints on the migration timescales.

This work benefited from support from PNP-CNRS and CS from Paris Observatory. The authors thank Paris Observatory SIO, and GENCI-CINES, for providing the necessary computational resources for this work.

\section{REFERENCES}

Boué, G., \& Laskar, J. 2006, Icarus, 185, 312

Boué, G., Laskar, J., \& Kuchynka, P. 2009, ApJL, 702, L19

Estrada, P. R., \& Mosqueira, I. 2006, Icarus, 181, 486

Goldreich, P. 1966, Reviews of Geophysics, 4, 411

Korycansky, D. G., Bodenheimer, P., Cassen, P., \& Pollack, J. B. 1990, Icarus, 84, 528

Laskar, J., \& Jacobson, R. A. 1987, A\&A, 188, 212

Laskar, J., \& Robutel, P. 1993, Nature, 361, 608

Lee, M. H., Peale, S. J., Pfahl, E., \& Ward, W. R. 2007, Icarus, 190, 103

Lykawka, P. S., Horner, J., Jones, B. W., \& Mukai, T. 2009, MNRAS, 1147

Morbidelli, A., Brasser, R., Tsiganis, K., Gomes, R., \& Levison, H. F. 2009, A\&A, 507, 1041

Mosqueira, I., \& Estrada, P. R. 2003a, Icarus, 163, 198

—. 2003b, Icarus, 163, 232

Murray-Clay, R. A., \& Chiang, E. I. 2005, ApJ, 619, 623

Tremaine, S. 1991, Icarus, 89, 85

Tsiganis, K., Gomes, R., Morbidelli, A., \& Levison, H. F. 2005, Nature, 435, 459

Ward, W. R. 1975, AJ, 80, 64

Ward, W. R., \& Hamilton, D. P. 2004, AJ, 128, 2501 


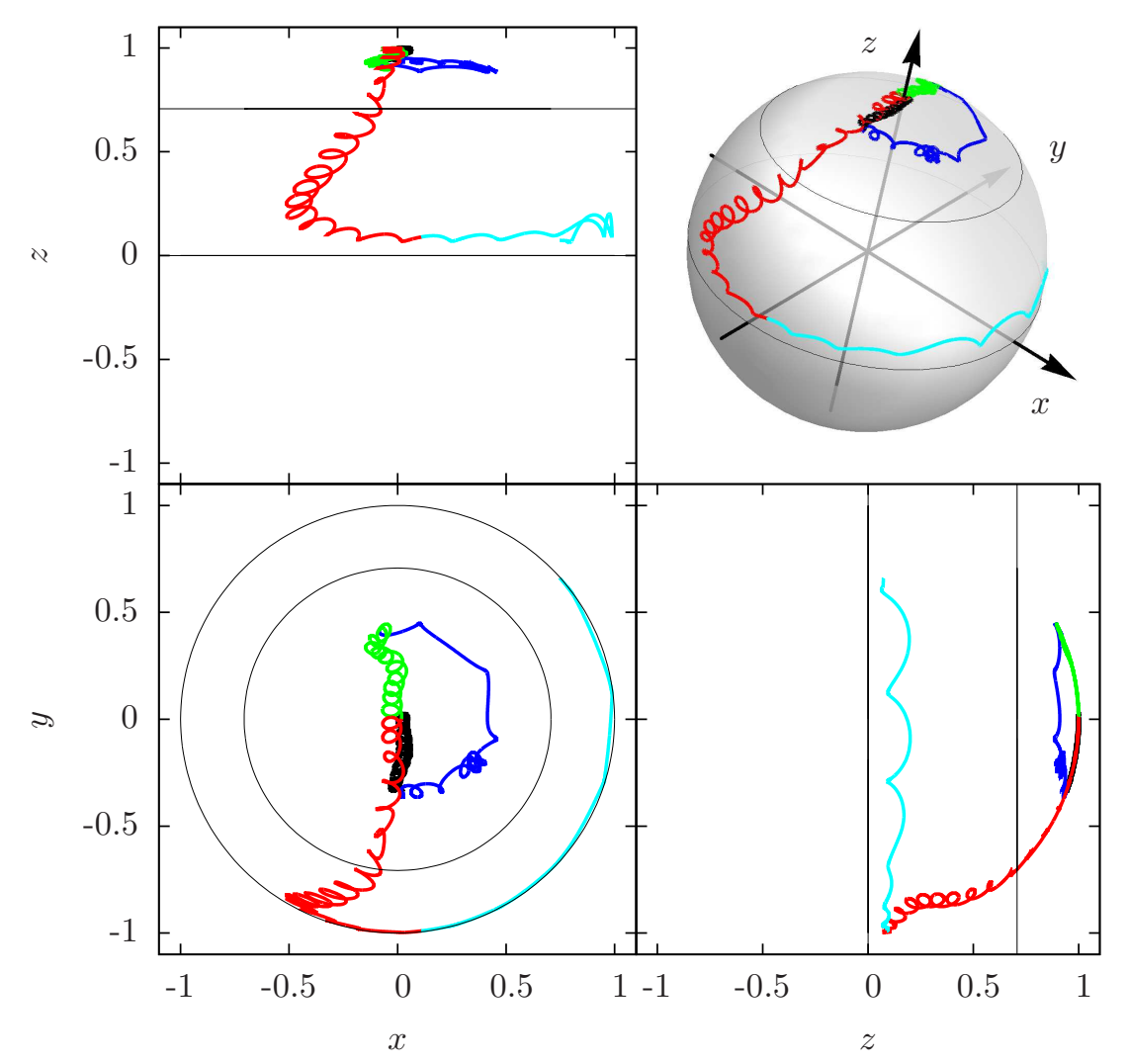

